21st Particles and Nuclei International Conference (PANIC 2017)

International Journal of Modern Physics: Conference Series

Vol. 46 (2018) 1860080 (6 pages)

(C) The Author(s)

DOI: $10.1142 / \mathrm{S} 2010194518600807$

\title{
Ring Imaging Cherenkov Detector Technologies for Particle Identification in the Electron-Ion Collider Experiments
}

\author{
X. He (for the EIC PID Consortium (eRD14 Collaboration)) \\ Department of Physics and Astronomy, Georgia State University, \\ 25 Park Place, Rm605, Atlanta, GA 30303, USA \\ xhe@gsu.edu
}

Published 3 May 2018

\begin{abstract}
In the proposed Electron-Ion Collider (EIC) experiments, particle identification (PID) of the final state hadrons in the semi-inclusive deep inelastic scattering allows the measurement of flavor-dependent gluon and quark distributions inside nucleons and nuclei. The EIC PID consortium (eRD14 Collaboration) has been formed for identifying and developing PID detectors using Ring Imaging Cherenkov (RICH) techniques for the EIC experiments. A modular Ring Imaging Cherenkov (mRICH) detector has been designed for particle identification in the momentum coverage from $3 \mathrm{GeV} / \mathrm{c}$ to $10 \mathrm{GeV} / \mathrm{c}$. The mRICH detector consists of an aerogel radiator block, a Fresnel lens, a mirror-wall and a photosensor plane. The first prototype of this detector was successfully tested at Fermi National Accelerator Laboratory in April 2016 for verifying the detector working principles. This talk will highlight the mRICH beam test results and their comparison with GEANT4-based detector simulations. An implementation of the mRICH detector concept in the Forward Angle sPHENIX spectrometer at BNL will also be mentioned in this talk.
\end{abstract}

Keywords: Electron-Ion Collider; Ring Imaging Cherenkov; RICH; GEANT4.

\section{Introduction}

The Electron-Ion Collider (EIC), which is supported in the 2015 Long Range Plan of the US Department of Energy for Nuclear Science ${ }^{1}$, aims to broaden the knowledge of nuclear structure by building nuclear tomography from quark and gluon distributions in multi-dimensional spaces such as spatial, momentum, spin, and flavor space ${ }^{2}$. One of the key requirements of the EIC experiments is to provide the particle identification (PID) capability of the final state hadrons from semi-inclusive deep inelastic scattering.

This is an Open Access article published by World Scientific Publishing Company. It is distributed under the terms of the Creative Commons Attribution 4.0 (CC-BY) License. Further distribution of this work is permitted, provided the original work is properly cited. 
X. He

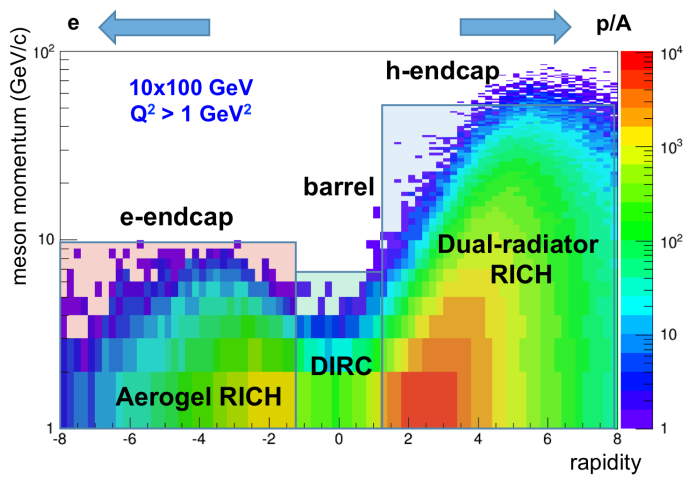

Fig. 1. Momentum vs rapidity distribution of final mesons from simulation of collisions of $10 \mathrm{GeV} / \mathrm{c}$ electron and $100 \mathrm{GeV} / \mathrm{c}$ proton, $Q^{2}>1 \mathrm{GeV}^{2}$.

Figure 1 shows the meson momentum distribution as a function of its rapidity which relates to the momentum direction of the meson ${ }^{2}$. In the hadron-going direction (called h-endcap, $1.5<$ rapidity $<8$ ) and the electron-going direction (called e-endcap, $-8<$ rapidity $<-1.5$ ), the meson momenta can reach $100 \mathrm{GeV} / \mathrm{c}$ and $10 \mathrm{GeV} / \mathrm{c}$, respectively. Along the barrel region $(-1.5<$ rapidity $<1.5)$, however, the momenta of the final state mesons are below $10 \mathrm{GeV} / \mathrm{c}$. To fulfill the different kinematics requirements and spacial limitations of the EIC experiments, three RICH-based detector designs for PID have been proposed by the EIC PID Consortium (eRD14), which include a dual-radiator $\mathrm{RICH}^{3}$, a high-performance $\mathrm{DIRC}^{4}$, and a modular RICH (mRICH).

The details of the first mRICH prototype have been published ${ }^{5}$. The highlights of this prototype design and the beam test results are given in section 2 and 3 . A new mRICH prototype has been built (see section 4) and it will be tested at Fermilab in late spring of 2018. At the same time, the conceptual implementations of an array of mRICH detectors in the Forward sPHENIX spectrometer and in the JLab EIC detector (called IP1 detector) are currently under study in GEANT4 simulation. A brief description of these implementations are given in section 5 .

\section{2. mRICH Detector Design}

In order to meet the challenge of the confined volume of electron endcap in the EIC experiments, a compact mRICH detector is proposed for $K / \pi$ separation up to $10 \mathrm{GeV} / \mathrm{c}$. At the same time, the mRICH design has a significant potential for $e / \pi$ identification providing an important capability supplementing the electromagnetic calorimeters and other possible $e / \pi$ ID systems. In an electron-ion collider, detection and identification of the scattered electron is a crucial capability. Here, a challenge is posed by the charged pion background, which rises rapidly at lower momenta. Thus, to access kinematics where the scattered electron has low momentum one needs a good pion suppression. As shown in Fig. 2, the first mRICH prototype consists of a 

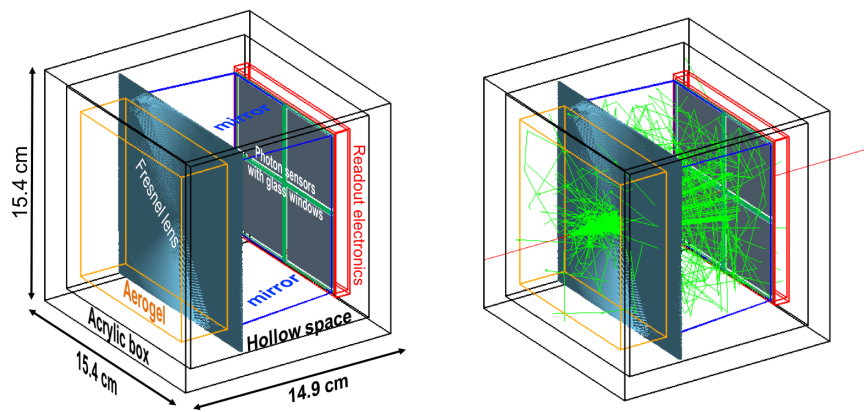

Fig. 2. (Left) mRICH detector and its components; (right) an event display of a single $9 \mathrm{GeV} / \mathrm{c}$ pion traveling toward to the center of the mRICH detector.
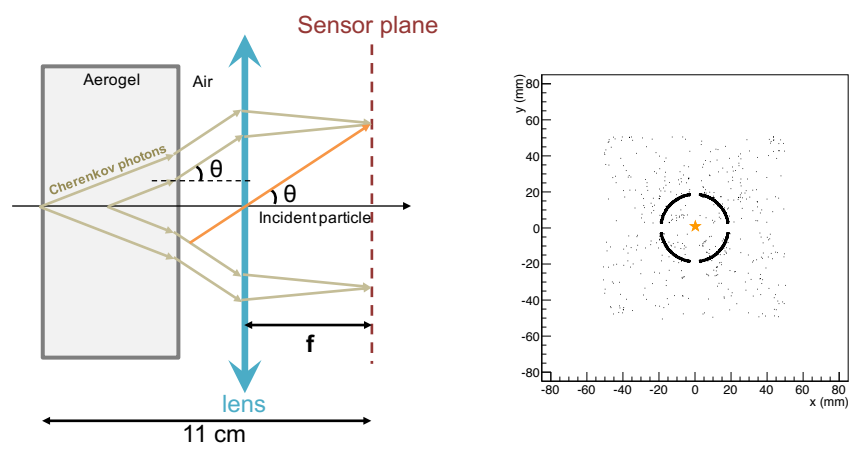

Fig. 3. (Left) Ray diagram showing the working principle of the mRICH detector. (Right) Ring image from 100 pions at $10 \mathrm{GeV} / \mathrm{c}$ generated from Geant4 simulation.

$3.3 \mathrm{~cm}$ thick block of aerogel with refractive index $n=1.03$ as a radiator, a Fresnel lens with focal length $f=3$ inch for image focusing, a four-sided mirror wall, and the focal surface covered by four H8500 multianode PMTs from Hamamatsu ${ }^{6}$ with pixel size of $6 \times 6 \mathrm{~mm}^{2}$.

There are two advantages of the lens-based design: (1) forming a sharper ring image in comparison with the proximity focusing RICH detector design ${ }^{7}$, as shown in Fig. 3; and (2) shifting the ring image by the lens to the central region of the focal surface as shown in Fig. 4, which results in a smaller required sensor plane area.

\section{3. mRICH Beam Test}

The first mRICH beam test was performed at Fermilab in April of 2016, which focused on the study of the detector working principle and the comparison with simulated results using GEANT4. The $120 \mathrm{GeV} / \mathrm{c}$ primary proton beam were incident perpendicular to the $\mathrm{mRICH}$ detector with a relatively small beam dispersion 
X. He
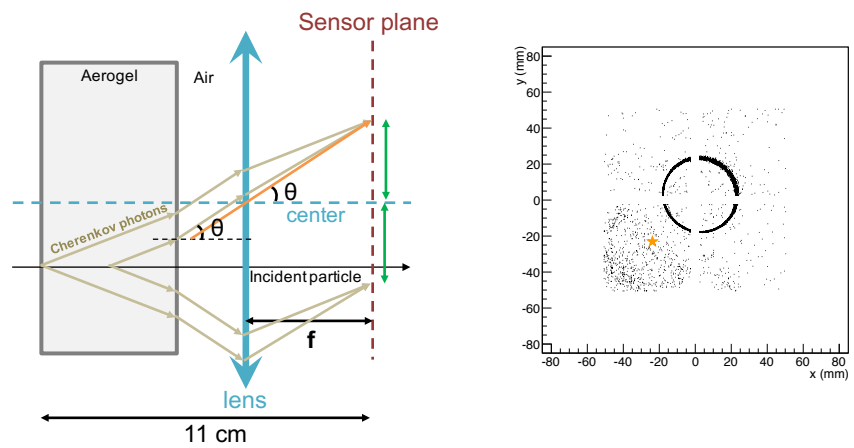

Fig. 4. (Left) Ray diagram showing the shifted ring image to the central region on the sensor plane. (Right) Ring image from 100 pions at $10 \mathrm{GeV} / \mathrm{c}$ formed at the central region on the sensor plane when the charged particles enter the detector off the principle axis of the lens.

Table 1. Summary of test beam data, analytical estimation and simulation from $120 \mathrm{GeV} / \mathrm{c}$ proton beam that enter at the center of the detector.

\begin{tabular}{lccc}
\hline & Estimation & Test Beam Data & Simulation \\
\hline Ring radius $(\mathrm{mm})$ & 19.4 & $19.0 \pm 1.3$ & $18.9 \pm 1.0$ \\
Total number of photons & 10.4 & $11.0 \pm 2.9$ & $11.1 \pm 2.9$ \\
\hline
\end{tabular}

in the transverse direction. The results are summarized in Table 1 together with estimations from analytical calculations and GEANT4 simulations; test results and estimations agree pretty well. The success of this beam test validated the concept of the mRICH detector design and established the baseline for the continued mRICH R\&D study.

\section{The Next mRICH Prototype}

The goal of the next mRICH prototype is to verify its PID performance with a few design improvements which include: (1) a longer focal length $(f=6 ")$ Fresnel lens;
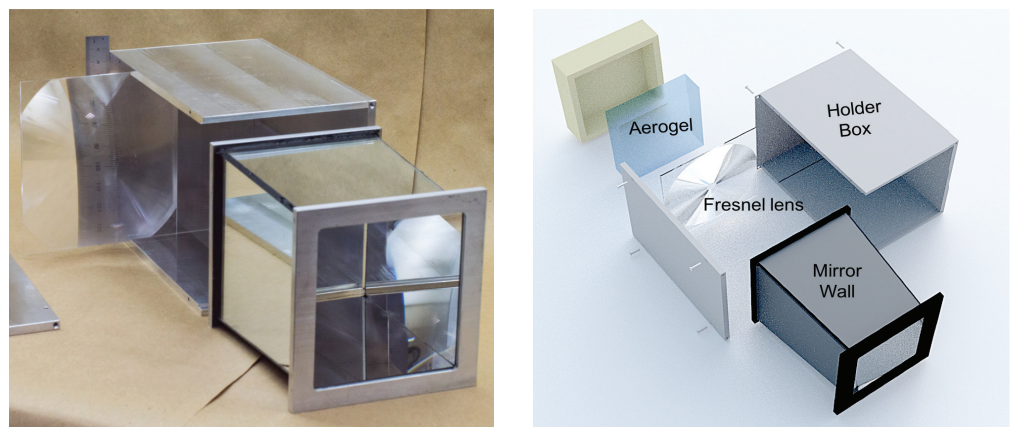

Fig. 5. (Left) The new mRICH prototype under construction; (Right) 3D illustration of the mRICH components. 


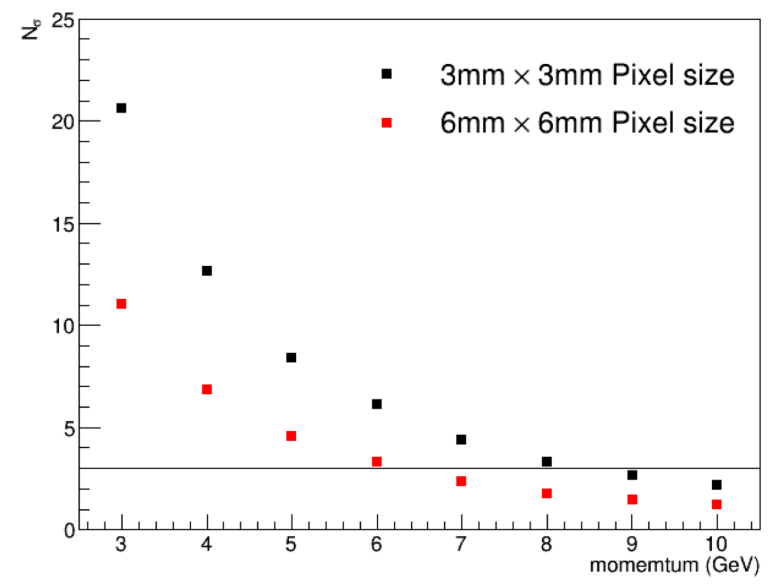

Fig. 6. $K / \pi$ separation power (number of sigma) versus incident particle momentum from simulation with 6 inch long focal length Fresnel lens, but with different resolution of photon sensors: $3 \times 3 \mathrm{~mm}^{2}$ (black), $6 \times 6 \mathrm{~mm}^{2}$ (red).

(2) photosensors of smaller pixel size $(3 \mathrm{~mm} \times 3 \mathrm{~mm})$ from Hamamatsu; and (3) new mRICH holder box design which separates the optical and readout components of mRICH. Figure 5 shows the new prototype under construction and a 3D illustration of the mRICH components.

The projected $K / \pi$ separation power based on the validated simulation for the new prototype is shown in Fig. 6 . With the smaller pixel size (i.e., $3 \mathrm{~mm} \times 3 \mathrm{~mm}$ ) of the photosensor, we are expecting to reach $3 \sigma$ separation power up to $8 \mathrm{GeV} / \mathrm{c}$.

\section{Summary and Outlook}

The EIC PID consortium has developed a suite of RICH detector technologies for particle identification in the future EIC experiments. This article focused on the EIC PID mRICH detector, the succesful proof of concept by the Fermilab beam test

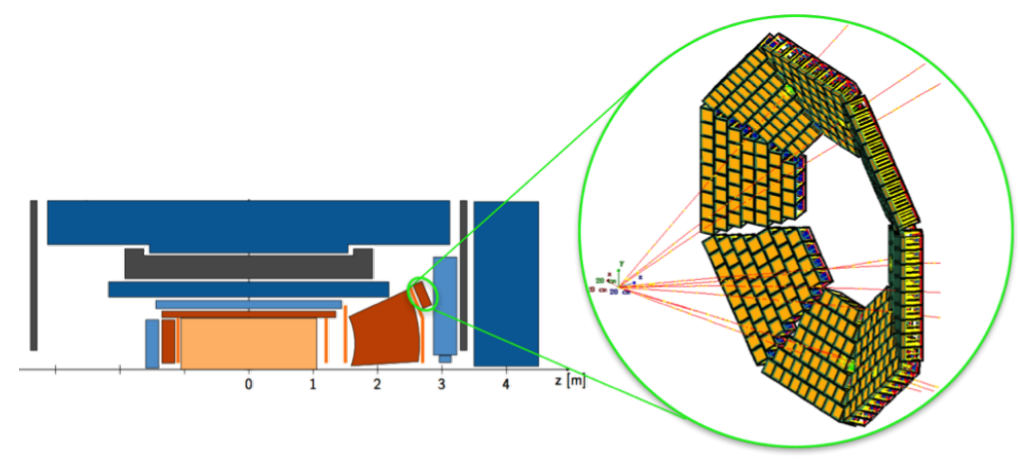

Fig. 7. An array of mRICH modules implemented in the Forward Angle sPHENIX experiment. 
X. He

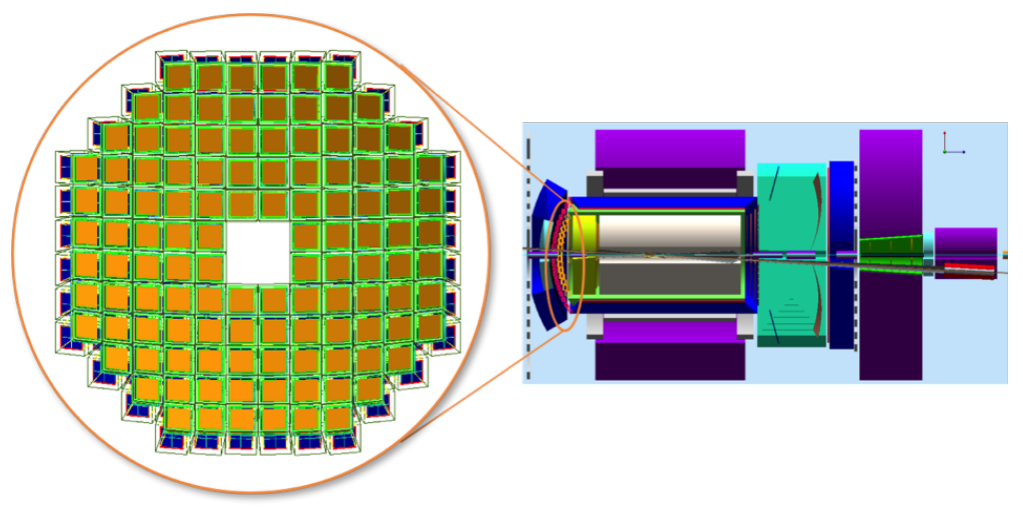

Fig. 8. An array of mRICH modules implemented in the conceptual design of the JLab EIC detector.

on its first prototype, and the design of the next prototype toward a detailed characterization of its PID capabilities. The excellent results of the mRICH prototype beam test, combined to the detector compactness and flexibility have stimulated the exploitation of mRICH arrays in the sPHENIX experiment; implementation of mRICH arrays in the simulations of the Forward Angle sPHENIX spectrometer (Fig. 7) and the JLab EIC detector concept (Fig. 8) is ongoing in order to study the detector performance in realistic experiments.

\section{Acknowledgments}

The author would like to thank the staff of the Fermilab Test Beam Facility for their assistance in carrying out the beam test of the first mRICH prototype. The author would also like to thank the members of the Instrument Shop of the Department of Physics and Astronomy at Georgia State University for machining the detector components of the mRICH prototype. This project was partially supported by the EIC detector R\&D fund which was managed by Brookhaven National Lab.

\section{References}

1. A. Aprahamian et al., Reaching for the horizon: The 2015 long range plan for nuclear science (2015).

2. A. Accardi et al., Eur. Phys. J. A 52, p. 268 (2016).

3. A. Del Dotto et al., Nucl. Instrum. Methods Phys. Res., Sect. A 876, 237 (2017).

4. G. Kalicy et al., J. Instrum. 11, p. C07015 (2016).

5. C. Wong et al., Nucl. Instrum. Methods Phys. Res., Sect. A 871, 13 (2017).

6. Flat planel type multianode pmt assembly H8500 series / H10966 series.

7. I. Adachi et al., IEEE Transactions on Nuclear Science 50, 1142 (Aug 2003). 